\title{
Spatiotemporal variation of groundwater quality using integrated multivariate statistical and geostatistical approaches in Amol-Babol Plain, Iran
}

\begin{abstract}
In recent years, groundwater quality has become a global concern due to its effect on human life and natural ecosystems. To assess the groundwater quality in the Amol-Babol Plain, a total of 308 water samples were collected during wet and dry seasons in 2009. The samples were analysed for their physico-chemical and biological constituents. Multivariate statistical analysis and geostatistical techniques were applied to assess the spatial and temporal variabilities of groundwater quality and to identify the main factors and sources of contamination. Principal component analysis (PCA) revealed that seven factors explained around $75 \%$ of the total variance, which highlighted salinity, hardness and biological pollution as the dominant factors affecting the groundwater quality in the Plain. Two-way analysis of variance (ANOVA) was conducted on the dataset to evaluate the spatio-temporal variation. The results showed that there were no significant temporal variations between the two seasons, which explained the similarity between six component factors in dry and wet seasons based on the PCA results. There are also significant spatial differences $(p>0.05)$ of the parameters under study, including salinity, potassium, sulphate and dissolved oxygen in the plain. The least significant difference (LSD) test revealed that groundwater salinity in the eastern region is significantly different to the central and western side of the study area. Finally, multivariate analysis and geostatistical techniques were combined as an effective method for demonstrating the spatial structure of multivariate spatial data. It was concluded that multiple natural processes and anthropogenic activities were the main sources of groundwater salinization, hardness and microbiological contamination of the study area.
\end{abstract}

Keyword: Groundwater quality; Statistical analysis; Two-way ANOVA; Geostatistical technique; Amol-Babol Iran 